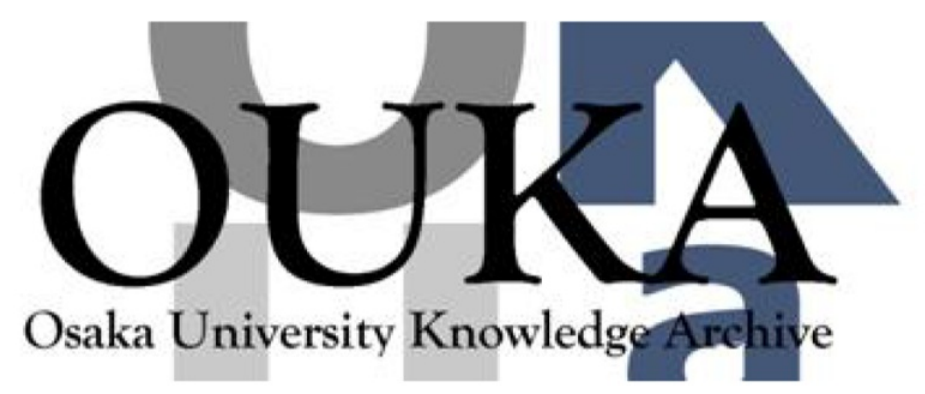

\begin{tabular}{|c|l|}
\hline Title & $\begin{array}{l}\text { Correlation between laser accelerated MeV } \\
\text { proton and electron beams using simple fluid } \\
\text { model for target normal sheath acceleration }\end{array}$ \\
\hline Author(s) & Tampo, M.; Awano, S.; Bolton, P.R. et al. \\
\hline Citation & Physics of Plasmas. 17(7) p. 073110 \\
\hline Issue Date & $2010-07$ \\
\hline oaire:version & VoR \\
\hline URL & https://hdl. handle. net/11094/2924 \\
\hline rights & \\
\hline Note & \\
\hline
\end{tabular}

Osaka University Knowledge Archive : OUKA

https://ir. Library. osaka-u. ac. jp/

Osaka University 


\title{
Correlation between laser accelerated MeV proton and electron beams using simple fluid model for target normal sheath acceleration
}

\author{
M. Tampo, ${ }^{1,2, a)}$ S. Awano, ${ }^{3}$ P. R. Bolton, ${ }^{1}$ K. Kondo,${ }^{1}$ K. Mima,${ }^{2}$ Y. Mori, ${ }^{4}$ H. Nakamura, ${ }^{3}$ \\ M. Nakatsutsumi, ${ }^{3}$ R. B. Stephens, ${ }^{5}$ K. A. Tanaka, ${ }^{2,3}$ T. Tanimoto, ${ }^{3}$ T. Yabuuchi, ${ }^{3}$ \\ and R. Kodama ${ }^{2,3}$ \\ ${ }^{1}$ Photo-Medical Research Center and Advanced Photon Research Center, JAEA, Kyoto 619-0215, Japan \\ ${ }^{2}$ Institute of Laser Engineering, Osaka University, Yamada-oka 2-6, Suita, Osaka 565-0871, Japan \\ ${ }^{3}$ Graduate School of Engineering, Osaka University, Yamada-oka 2-1, Suita, Osaka 565-0871, Japan \\ ${ }^{4}$ The Graduate School for The Creation of New Photonics Industries, Kurematsu-cho 1955-1, \\ Hamamatsu City, Shizuoka, Japan \\ ${ }^{5}$ General Atomics, 3550 General Atomics Court, San Diego, California 92121-1122, USA
}

(Received 11 December 2009; accepted 11 June 2010; published online 29 July 2010)

\begin{abstract}
High density energetic electrons that are created by intense laser plasma interactions drive $\mathrm{MeV}$ proton acceleration. The correlation between accelerated $\mathrm{MeV}$ protons and escaped electrons is experimentally investigated at laser intensities in the range of $10^{18}-10^{19} \mathrm{~W} / \mathrm{cm}^{2}$ with $S$-polarization. Observed proton maximum energies are linearly proportional to escaped electron slope temperatures with a scaling coefficient of about 10. In the context of the simple analytical fluid model for transverse normal sheath acceleration, hot electron sheath density near the target rear surface can be estimated if an empirical acceleration time is assumed. (C) 2010 American Institute of Physics. [doi:10.1063/1.3459063]
\end{abstract}

\section{INTRODUCTION}

The study of proton acceleration to multi-MeV kinetic energies from plasmas produced by the interaction with an intense laser pulse at high intensity is a rapidly growing research area. This emergent proton beam is highly laminar; yet the emission typically exhibits a significant angular divergence about a central axis that is nearly parallel to the normal of the target rear surface. Such particle sources are interesting, in part because they have potential for important applications to proton acceleration, ${ }^{1}$ material studies, ${ }^{2}$ proton radiography, ${ }^{3}$ the possible production of short-lived radioisotopes for medical applications, and ion beam radiotherapy. ${ }^{4}$ However, laser-driven sources are not yet optimized for such applications and ultraintense laser plasma interactions relevant to proton beam generation are not yet fully understood. Because protons are accelerated in an electrostatic sheath field that is built up by the hot electrons in intense laser plasma interactions, it is important to study the relation between energetic proton and electron yields in order to optimize the proton beam generation and even to inspire diagnostics that can be correlated with proton yields. The electrostatic field is induced by a high density, hot electron sheath acting on a proton layer adsorbed as hydrogen impurities on the rear surface of a solid planar target. The electron sheath is originated in an intense electron beam, which is created during laser plasma interaction at the target front surface and transported through the target to the target rear side. A simple analytical fluid model describes the following dependence of the electrostatic sheath field on the sheath temperature $T_{e}$ and density $n_{e}$ at the target rear surface:

\footnotetext{
${ }^{a)}$ Electronic mail: tampo.motonobu@jaea.go.jp.
}

$$
E_{\text {field }}=\sqrt{\frac{n_{e} T_{e} q^{2}}{\epsilon_{0}}}
$$

where $q$ and $\epsilon_{0}$ are elementary charge and vacuum permittivity, respectively. ${ }^{5}$ When the protons are accelerated by the electrostatic field predicted by Eq. (1), the proton maximum energy can be predicted by using the collisionless fluid equations with a pressure term of the electrostatic field as follows: ${ }^{6}$

$$
E_{\max }=2 T_{e}\left(\ln \left\{\left[\frac{\omega_{p}\left(n_{e}\right) \Delta t}{\sqrt{2 e}}\right]+\sqrt{1+\left[\frac{\omega_{p}\left(n_{e}\right) \Delta t}{\sqrt{2 e}}\right]^{2}}\right\}\right)^{2},
$$

where $\omega_{p}=\left(n_{e} q^{2} / m_{i} \epsilon_{0}\right)^{1 / 2}$ is the ion plasma frequency, $\Delta t$ is the proton acceleration time interval, $m_{i}$ is the ion (proton) mass, and $e$ is the numerical constant equal to $2.71828 \ldots$. According to this simple model, for a specified proton acceleration time $(\Delta t)$, the proton maximum energy $\left(E_{\max }\right)$ depends only on two electron plasma parameters: the hot electron sheath temperature $T_{e}$ and its density $n_{e}$. However, these quantities have not been investigated simultaneously with proton beam measurements. This paper presents simultaneous measurements of proton spectra and escaped electron energy spectra from a target rear surface that can therefore be correlated. We have also measured source sizes of the proton beam and the proton areal density at the target rear surface using two-dimensional (2D) ion detection with energy resolution. The maximum proton energy and areal density of the proton beam are observed to depend on the escaped electron temperature $T_{\mathrm{es}}$. 


\section{EXPERIMENTAL CONDITION}

Experiments were conducted with two different $1053 \mathrm{~nm}$ laser systems at the Institute of Laser Engineering at Osaka University: the GEKKO Module 2 (GM2) (Ref. 7) and the GEKKO PW, ${ }^{8}$ both having an optical parametric chirped pulse amplification front end system. ${ }^{9}$ With the GM2 system we varied the laser pulse energy from 2 to $10 \mathrm{~J}$ with an average pulse duration of $600 \mathrm{fs}$. Pulse energy ranged from 80 to $100 \mathrm{~J}$ with a duration of $700 \mathrm{fs}$ for the GEKKO PW system. Laser light was focused onto planar Al targets with thickness of $10 \mu \mathrm{m}$. A grating structure was imprinted on the rear surface of the $\mathrm{Al}$ target to enable measurement of the proton source size. ${ }^{10}$ A 500 groove $/ \mathrm{mm}$ density was used with a peak-to-valley depth of $1 \mu \mathrm{m}$. With a $25^{\circ}$ angle of incidence and $S$-polarization of the laser field, the $f$-numbers of the focusing laser optics were 3 (at GM2) and 7 (at PW). The laser focal spot was monitored with an $\mathrm{x}$-ray pinhole camera using a charge coupled device detector. The spotsize (full width at half maximum) was varied from 30 to $50 \mu \mathrm{m}$ such that the laser intensities ranged from $1 \times 10^{18}$ to $1 \times 10^{19} \mathrm{~W} / \mathrm{cm}^{2}$.

An electron spectrometer (with a $5 \mathrm{~mm}$ collimator) was placed $580 \mathrm{~mm}$ downstream from the target rear surface in the direction parallel to that of laser propagation to measure the electrons accelerated by the laser ponderomotive force that escapes the sheath. ${ }^{11}$ Through the collimator accelerated electrons entered the magnetic field region of the spectrometer with a length of $240 \mathrm{~mm}$. The electron beam was deflected by the static magnetic field of $4200 \mathrm{G}$. The deflected electrons were recorded on an imaging plate (IP), which is a type of SR2025, and the recorded signal was scanned with an IP reader BAS 1800 (IP and the IP reader were provided by Fuji Film Co. Ltd.). To prevent high energy electrons or nondispersed electrons from directly hitting the IP the IP surface normal was perpendicular to the electron beam axis.

A Thomson parabola for recording proton energy spectra was placed $580 \mathrm{~mm}$ downstream of the target rear surface in the direction $10^{\circ}$ off target normal ${ }^{12}$ and used a $230 \mu \mathrm{m}$ diameter collimator. On passing through the collimator aperture protons were then dispersed by a static magnetic $(0.2 \mathrm{~T})$ and electric $(3 \mathrm{kV} / \mathrm{cm})$ fields within a region with length of $150 \mathrm{~mm}$. Because increased distance from the end of the field region to the track detector provides a higher energy resolution for high energy protons, the dispersed proton beam was recorded on a track detector that was placed 128 $\mathrm{mm}$ from an end of the static magnetic and electric field region. The resultant energy resolution ranged from $10 \mathrm{keV}$ for $0.6 \mathrm{MeV}$ protons to $1 \mathrm{MeV}$ for $20 \mathrm{MeV}$ protons. We used a solid state track detector (CR-39) which is insensitive to electrons and $\mathrm{x}$-rays. ${ }^{13}$ Because the Thomson parabola detector was required to measure broad proton energy spectra, we used HARZLAS TNF-1 (provided by the Fukubi Chemical Co. Ltd.), which could detect proton tracks from $100 \mathrm{keV}$ to $27 \mathrm{MeV}$ (Ref. 14) using an etching condition of $70{ }^{\circ} \mathrm{C}, 6 \mathrm{~N}$ $\mathrm{KOH}$ for $2 \mathrm{~h}$.

Between the target and the spectrometers a multilayered detector consisting of CR-39 of BARYO TRACK (provided by the Fukubi Chemical Co. Ltd.) and radio chromic film

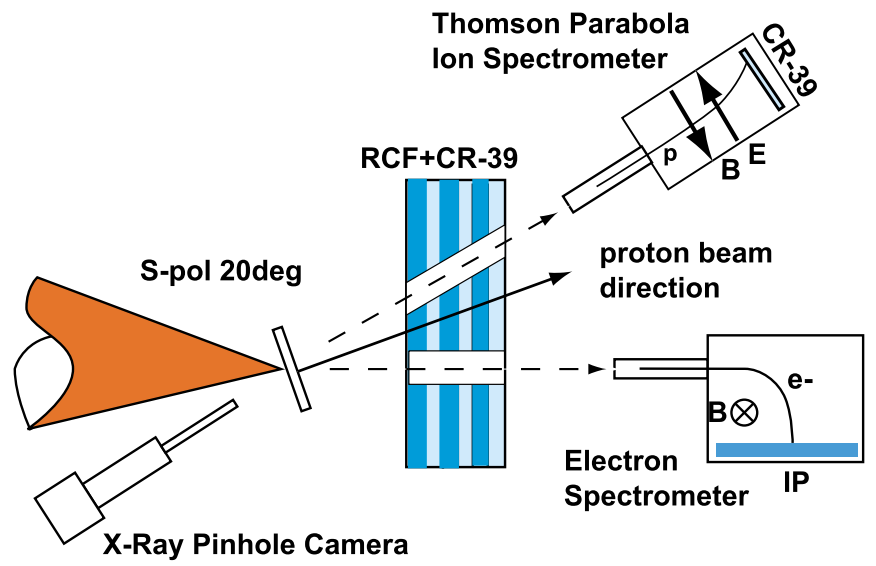

FIG. 1. (Color online) Sketch of experimental setup.

(RCF) of GAF Chromic HD810 (provided by the ISP Co. Ltd.) was used to measure the spatial distribution of the proton beam and to resolve proton energies up to the maximum energy. Two types of the CR-39 (BARYO TRACK) were selected to record proton tracks with the diameter of 1-6 $\mu \mathrm{m}$ (with an etching condition of $70{ }^{\circ} \mathrm{C}, 6 N \mathrm{KOH}$ for $0.5 \mathrm{~h}) .{ }^{15}$ One is of thickness $100 \mu \mathrm{m}$ and the other of thickness $400 \mu \mathrm{m}$. The RCF is one of the $2 \mathrm{D}$ dosimeter films used to measure the spatial distribution of protons. ${ }^{12}$ The dose is proportional to the proton-induced optical density change (i.e., from transparent to dark blue) in the sensitive dye layer of the RCF. For example, 1 Gy corresponds to an OD3 optical density (670 nm light). The RCF (HD810) had a sensitive layer of $5 \mu \mathrm{m}$ thickness and a polyester layer of $95 \mu \mathrm{m}$ thickness. ${ }^{16}$ The proton detector consisted of four kinds of multilayered films. The first layer was an $\mathrm{Al}$ foil with thickness of $12 \mu \mathrm{m}$, the second layer was three pieces of the RCF, the third layer was five pieces of CR-39 (100 $\mu \mathrm{m}$ thickness) and RCF, and then the fourth layer was four pieces of CR-39 (400 $\mu$ m thickness) and RCF. The second layer was sensitive to protons with energies exceeding $>1 \mathrm{MeV}$ and the third layer was sensitive to protons with energies exceeding $>6 \mathrm{MeV}$. The fourth layer was sensitive to those protons with energies in the range of $12-25 \mathrm{MeV}$. All pieces of the CR-39 had an energy resolution of $1 \mathrm{MeV}$ as determined by the track diameters. RCF films had energy resolutions from 1 to $4 \mathrm{MeV}$, approximately. ${ }^{12}$ This proton detector stack, of transverse dimensions $5 \times 5 \mathrm{~cm}^{2}$, was placed between the target and the spectrometers, $30 \mathrm{~mm}$ downstream of the target rear surface in the direction of the target normal. The detector stack also featured bored channels of $5 \mathrm{~mm}$ diameter leading to the Thomson parabola and electron spectrometer axes. Consequently electrons and protons coming from the target could be transported to the appropriate spectrometer without scattering in the stack detector as shown in Fig. 1.

\section{EXPERIMENTAL RESULTS}

Figure 2(a) reveals typical energy spectra of escaped electrons obtained at two different intensities: $4 \times 10^{18}$ and $1 \times 10^{19} \mathrm{~W} / \mathrm{cm}^{2}$. Assuming the electron spectra to have a Boltzmann distribution $\left[\exp \left(-E / T_{\mathrm{es}}\right)\right]$, the spectrum at 

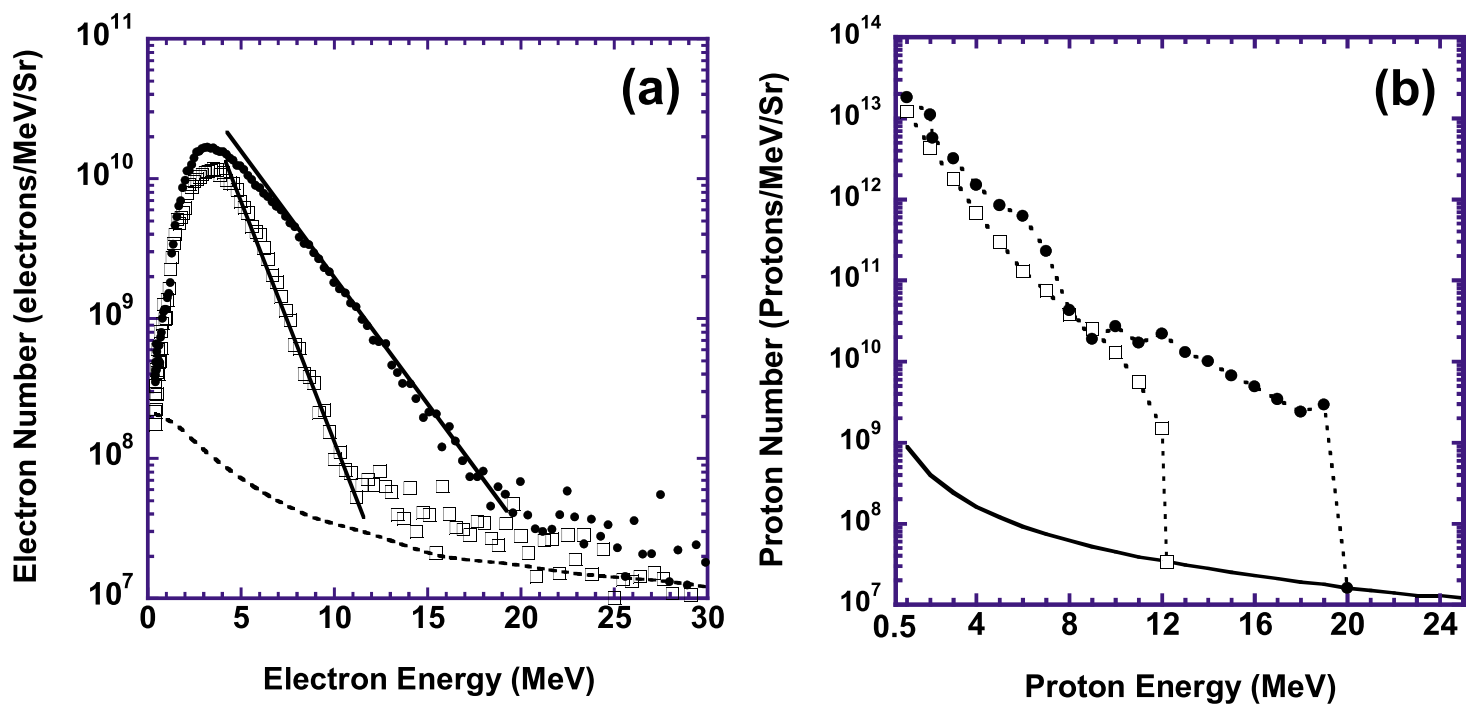

FIG. 2. (Color online) Energy spectra of escaped electron and proton beams obtained simultaneously using the Thomson parabola ion spectrometer and the magnetic electron spectrometer at different laser intensities. (a) Electron energy spectra shown with open rectangles and black dots are obtained at the 4 $\times 10^{18}$ and $1 \times 10^{19} \mathrm{~W} / \mathrm{cm}^{2}$, which can be fitted with the slope temperatures of $T_{\mathrm{es}}=1.1 \mathrm{MeV}$ and $T_{\mathrm{es}}=2.3 \mathrm{MeV}$, respectively, for Boltzmann distributions. The dashed line shows the detection limit. (b) Proton energy spectra obtained simultaneously with the escaped electron energy spectra of (a). The data points of open rectangles and black dots correspond to the data points at $T_{\mathrm{es}}=1.1 \mathrm{MeV}$ and $T_{\mathrm{es}}=2.3 \mathrm{MeV}$, respectively, of (a). The proton energy spectra have the spectral cutoffs at maximum energies of $E_{\max }=12 \mathrm{MeV}$ and $E_{\max }=20 \mathrm{MeV}$, respectively. The black solid line shows the detection limit.

$4 \times 10^{18} \mathrm{~W} / \mathrm{cm}^{2}$ as shown in Fig. 2(a) is fitted with a temperature of $1.1 \mathrm{MeV}$. The electron spectrum at $1 \times 10^{19} \mathrm{~W} / \mathrm{cm}^{2}$ as shown in Fig. 2(a) is fitted to the distribution with a $2.3 \mathrm{MeV}$ temperature. Figure 2(b) shows proton energy spectra observed simultaneously with electron spectra at the same two different intensities. The proton spectrum at $4 \times 10^{18} \mathrm{~W} / \mathrm{cm}^{2}$ as shown in Fig. 2(b) has a maximum proton energy $E_{\max }$ of $11 \mathrm{MeV}$ (spectral cutoff). The $E_{\text {max }}$ for the proton energy spectrum at $1 \times 10^{19} \mathrm{~W} / \mathrm{cm}^{2}$ as shown in Fig. 2(b) is $20 \mathrm{MeV}$. These results indicate a linear correlation between proton maximum energies and escaped electron temperatures.

Small errors in the placement of the Thomson parabola relative to the irradiation site on the target can result in misinterpretations of maximum proton energy; especially with narrower proton spatial distributions. Consequently maximum proton energies are evaluated from the proton detector stack that subtends a large detection solid angle. Furthermore the detector stack can also resolve angular distributions (i.e., spatial distributions) with resolution set in $1 \mathrm{MeV}$ steps. Using the proton detector stack and the electron spectrometer, the correlation between escaped electron slope temperature and maximum proton energy was obtained by changing the laser intensities as shown Fig. 3. The proton maximum en$\operatorname{ergy}\left(E_{\max }\right)$ is proportional to the electron slope temperatures $\left(T_{\mathrm{es}}\right)$ according to the linear relation, $E_{\max } \sim 10 T_{\mathrm{es}}$ with a $10 \%$ error. Protons are accelerated by the electrostatic sheath field generated between the high density electron cloud and the proton layer adsorbed in the target rear surface. Spatial proton distributions were obtained with the CR-39 and the RCF in the proton detector stack. Figure. 4(a) illustrates a typical image of the proton beam observed with the RCF. The optical density of the proton beam image shown with a grayscale is proportional to proton dose. Because the proton doses are also proportional to the proton areal densities, the proton beam image on the RCF shown in Fig. 4(a) corresponds to a spatial distribution of proton densities. As shown in Fig. 4(a), a pattern of stripes is superimposed in the image. The stripe pattern shows a periodic variation of optical density on the gray scale. Therefore the spatial proton density distribution includes periodic proton density fluctuations. The proton density profile including these fluctuations is shown in Fig. 4(b). The periodic density fluctuation is gen-

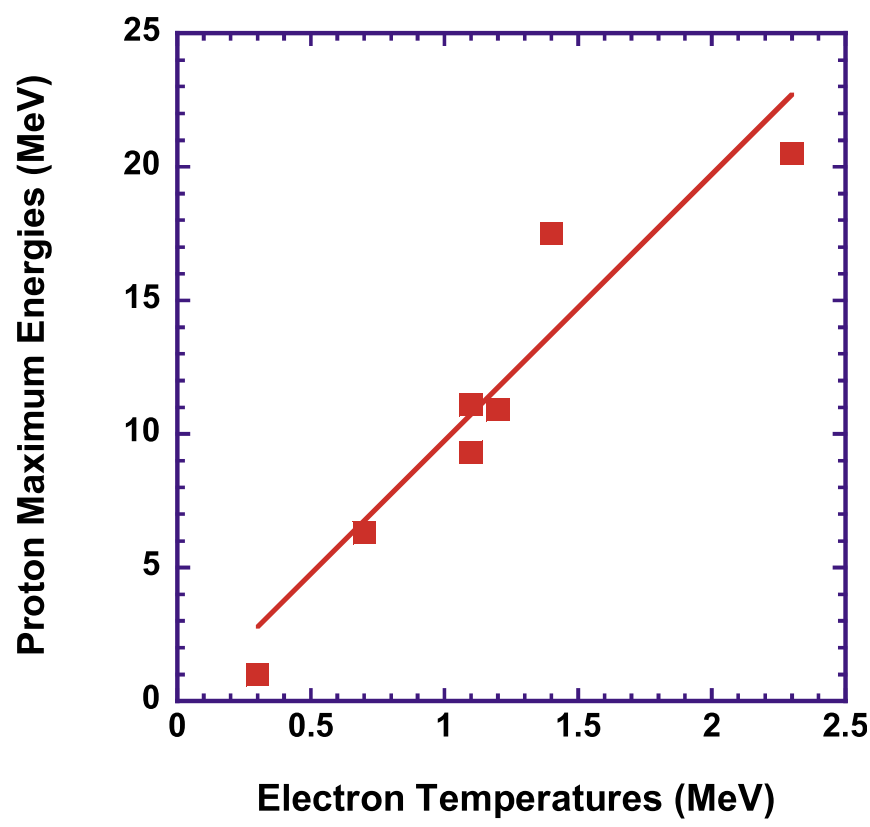

FIG. 3. (Color online) Correlation between escaped electron temperature $\left(T_{\mathrm{es}}\right)$ and proton maximum energy $\left(E_{\mathrm{max}}\right)$. Data points (rectangles) are fitted with the interpolation curve of $E_{\max }=10 T_{\mathrm{es}}$. 


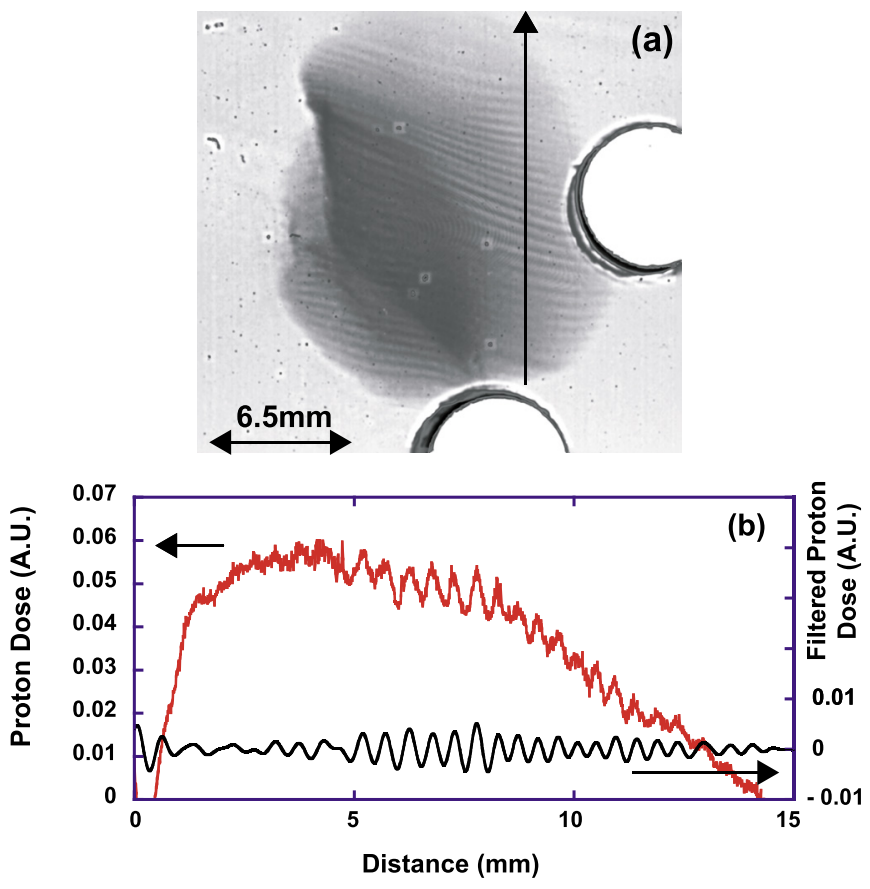

FIG. 4. (Color online) (a) Proton beam image on RCF with a periodic modulation of the signal intensities affected by the grating structures on the target rear surface. (b) Proton dose profile along the line shown in proton image of (a) and periodic density modulation filtered from the proton dose profile. The peak number of the periodic density peaks is 25 .

erated from the grating structure that is imprinted in the target rear surface. ${ }^{10}$ The number of peaks in this modulation of the spatial proton distribution corresponds to the number of grating groves on the target rear surface that are included within the proton source region. Therefore by knowing the number of modulation peaks we know the source size which is simply the number of groves times $2 \mu \mathrm{m}$. According to the number of modulation peaks the number of grating grooves in the source region of the proton beam, as shown in Fig. 4(a), is 25 in Fig. 4(b). The proton beam image in Fig. 4(a) also shows protons with energy $E>15 \mathrm{MeV}$. Consequently we determine that the proton beam of this energy is generated from a source area with a diameter of $50 \mu \mathrm{m}$. The areal density of the protons accelerated to $15 \mathrm{MeV}$ can be also evaluated by dividing the proton number, as given by the spatial distribution of proton beam, by the proton source area. The total proton number is evaluated from the CR-39 recording proton tracks with $16 \mathrm{MeV}$, which is a layer next to the RCF shown in Fig. 4(a) and also have almost same beam size as Fig. 4(a). The total proton number is determined to be $1.6 \times 10^{8}$ protons. The areal density of the protons accelerated to $15 \mathrm{MeV}$ is accordingly estimated to be $8.1 \times 10^{12}$ protons $/ \mathrm{cm}^{2}$.

We conducted experiments using the grating targets irradiated with the ultraintense laser pulse delivered from Gekko PW, with varying laser peak intensity due to spotsize variation but the same pulse energy at $100 \mathrm{~J}$ for each shot. Since each pulse laser irradiation of the grating target generates both electron and proton beams, we can demonstrate the correlation between the escaped electron slope temperature and the maximum proton energy as listed in Table I. In the proton
TABLE I. Correlation of escaped electron slope temperature with proton source size and areal density at the maximum proton energy obtained for a laser pulse energy $100 \mathrm{~J}$.

\begin{tabular}{lccc}
\hline \hline $\begin{array}{l}T_{e} \\
(\mathrm{MeV})\end{array}$ & $\begin{array}{c}E_{\max } \\
(\mathrm{MeV})\end{array}$ & $\begin{array}{c}\text { Proton source size } \\
\left(\mathrm{cm}^{2}\right)\end{array}$ & $\begin{array}{c}\text { Proton areal density } \\
\left(\text { protons } / \mathrm{cm}^{2}\right)\end{array}$ \\
\hline 1.4 & 18 & $7.0 \times 10^{-6}$ & $1.1 \times 10^{13}$ \\
2.3 & 21 & $3.1 \times 10^{-6}$ & $5.3 \times 10^{12}$ \\
\hline \hline
\end{tabular}

beam images (corresponding to the maximum energies in Table I) there are periodical proton density fluctuations as seen in the proton beam image of Fig. 4(a). Based on this target grating structure we determined the source size of proton beams with maximum energies as shown in Table I. It can be seen that the source size of the proton beams listed in Table I decreases with increased escaped electron temperature and maximum proton energy. These experimentally determined areas correspond to spotsizes of 30 and $20 \mu \mathrm{m}$ with an assumed circular focal spot for electron temperatures of 1.4 and $2.3 \mathrm{MeV}$, respectively. Using the proton source sizes and proton numbers from the CR-39 recording proton tracks, areal densities of protons accelerated to these maximum energies can also be determined. Table I indicates that the areal densities also decrease with increased escaped electron temperature and with increased maximum proton energy.

\section{DISCUSSION}

As shown in the table the spotsize reduction for the 2.3 MeV electron temperature relative to that at $1.4 \mathrm{MeV}$ corresponds to a laser intensity increase for the fixed $100 \mathrm{~J}$ pulse energy and the maximum proton energy scales approximately as the square root of this intensity as expected in the target normal sheath acceleration (TNSA) regime. Because it is correlated with the maximum proton energy, the measured slope temperature of the escaped electrons, which can be higher than the electron sheath temperature, also scales with the square root of the laser intensity.

In addition to this temperature assumption we also use an empirical expression for effective acceleration time $t_{\text {acc }}$ as determined from the laser pulse duration $t_{\text {laser }}$ $\left(t_{\text {acc }} \sim 1.3 \times t_{\text {laser }}\right) .{ }^{17}$ According to Eq. (2) the hot electron sheath density is a decreasing function of the ratio $E_{\max } / T_{e}$, where $T_{e}$ is the sheath temperature. Therefore, using the empirical value for acceleration time and the escaped electron temperature, $T_{\mathrm{es}}$ in Eq. (2) can provide a lower limit estimate for the hot electron sheath density. In this way, using Table I temperatures, we obtain limiting hot electron densities, $n_{e}$ of $1.4 \times 10^{20} \mathrm{~cm}^{-3}$ (at $T_{e}=1.4 \mathrm{MeV}$ and maximum proton energy of $18 \mathrm{MeV}$ ), and $6.2 \times 10^{19} \mathrm{~cm}^{-3}$ (at $T_{e}=2.3 \mathrm{MeV}$ and maximum proton energy of $21 \mathrm{MeV}$ ).

As shown in Table I the proton areal densities apply to the tabulated maximum energies and are of order $10^{12}-10^{13}$ protons $/ \mathrm{cm}^{2}$. These multi-MeV protons are generated from the rear surface of the $\mathrm{Al}$ target and originate from hydrogen molecules that have been adsorbed on the target rear surface as water or oil. ${ }^{18,19}$ If we assume that 
surface protons are trapped within an average depth near $100 \mathrm{~nm}$, moving around with a mean adsorption time of $10^{-13} \mathrm{~s},{ }^{20}$ then we can estimate the time-averaged proton density by dividing the observed areal density by this effective depth of hydrogen adsorption. ${ }^{21,22}$ Accordingly the proton densities at the maximum energies of 18 and $21 \mathrm{MeV}$ are estimated to be $1.1 \times 10^{18}$ and $5.3 \times 10^{17} \mathrm{~cm}^{-3}$, respectively. As expected these energy specific densities are significantly lower than the hot electron sheath density limits determined using escaped electron temperatures in Eq. (2).

\section{SUMMARY}

We have simultaneously measured energy spectra of electron and proton beams generated by intense laser irradiation from the rear side of thin Al foil targets, which have imprinted grating structures. With varying laser intensities, we have shown explicitly that proton maximum energies and escaped electron slope temperatures are linearly correlated according to the relation, $E_{\mathrm{max}}=10 T_{\mathrm{es}}$. From measured spatial proton distributions we have also evaluated the proton source sizes and proton areal density. Energy specific proton densities are also estimated based on the depth of hydrogen absorption at the target rear surface. Limiting hot electron densities near the target rear surface have been determined using the simple fluid model for TNSA regime assuming the empirical estimate for "effective" ion acceleration time and the escaped electron slope temperature. With this methodology improved hot electron sheath density estimates can be made if the sheath temperature is known.

\section{ACKNOWLEDGMENTS}

The authors thank ILE GOD group for their operation of the GX2 and GM2 Laser system, and TM group for their fabrication of targets.

This work was partially supported by MEXT, a Grantin-Aid for Creative Scientific Research (Grant No. 15GS0214).

${ }^{1}$ J. M. de Conto, J. Phys. (Paris) IV 09, Pr7-115 (1999).

${ }^{2}$ D. S. Gemmell, Rev. Mod. Phys. 46, 129 (1974).

${ }^{3}$ N. S. P. King, E. Ablesb, K. Adams, K. R. Alrick, J. F. Amann, S. Balzar, P. D. Barnes, Jr., M. L. Crow, S. B. Cushing, J. C. Eddleman, T. T. Fife, P.
Flores, D. Fujino, R. A. Gallegos, N. T. Gray, E. P. Hartouni, G. E. Hogan, V. H. Holmes, S. A. Jaramillo, J. N. Knudsson, R. K. London, R. R. Lopez, T. E. McDonald, J. B. McClelland, F. E. Merrill, K. B. Morley, C. L. Morris, F. J. Naivar, E. L. Parker, H. S. Park, P. D. Pazuchanics, C. Pillai, C. M. Riedel, J. S. Sarracino, F. E. Shelley, Jr., H. L. Stacya, B. E. Takala, R. Thompson, H. E. Tucker, G. J. Yates, H. -J. Ziock, and J. D. Zumbro, Nucl. Instrum. Methods Phys. Res. A 424, 84 (1999).

${ }^{4}$ J. Raloff, Sci. News (Washington, D. C.) 156, 257 (1999).

${ }^{5}$ J. E. Crow, P. Auer, and J. E. Allen, J. Plasma Phys. 14, 65 (1975).

${ }^{6}$ P. Mora, Phys. Rev. Lett. 90, 185002 (2003).

${ }^{7}$ M. Mori, Y. Kitagawa, R. Kodama, K. Sawai, and S. Nakai, Technol. Rep. Osaka Univ. 49, 139 (1999).

${ }^{8}$ Y. Kitagawa, H. Fujita, R. Kodama, H. Yoshida, S. Matsuo, T. Jitsuno, T. Kawasaki, H. Kitamura, T. Kanabe, S. Sakabe, K. Shigemori, N. Miyanaga, and Y. Izawa, IEEE J. Quantum Electron. 40, 281 (2004).

${ }^{9}$ H. Yoshida, E. Ishii, R. Kodama, H. Fujita, Y. Kitagawa, Y. Izawa, and T. Yamanaka, Opt. Lett. 28, 257 (2003).

${ }^{10}$ T. E. Cowan, J. Fuchs, H. Ruhl, A. Kemp, P. Audebert, M. Roth, R. Stephens, I. Barton, A. Blazevic, E. Brambrink, J. Cobble, J. Fernandez, J. C. Gauthier, M. Geissel, M. Hegelich, J. Kaae, S. Karsch, G. P. Le Sage, S. Letzring, M. Manclossi, S. Meyroneinc, A. Newkirk, H. Pepin, and N. Renard-LeGalloudec, Phys. Rev. Lett. 92, 204801 (2004).

${ }^{11}$ K. A. Tanaka, T. Yabuuchi, T. Sato, R. Kodama, Y. Kitagawa, T. Takahashi, T. Ikeda, Y. Honda, and S. Okuda, Rev. Sci. Instrum. 76, 013507 (2005).

${ }^{12}$ R. A. Snavely, M. H. Key, S. P. Hatchett, T. E. Cowan, M. Roth, T. W. Phillips, M. A. Stoyer, E. A. Henry, T. C. Sangster, M. S. Singh, S. C. Wilks, A. MacKinnon, A. Offenberger, D. M. Pennington, K. Yasuike, A. B. Langdon, B. F. Lasinski, J. Johnson, M. D. Perry, and E. M. Campbell, Phys. Rev. Lett. 85, 2945 (2000).

${ }^{13}$ S. A. Durrani and R. K. Bull, Solid State Nuclear Track Detection: Principles, Methods, and Applications (International Series in Natural Philosophy) (Pergamon, New York, 1985), Vol. 111.

${ }^{14}$ K. Ogura, M. Asano, N. Yasuda, and M. Yoshida, Nucl. Instrum. Methods Phys. Res. B 185, 222 (2001).

${ }^{15}$ T. Yamauchi, T. Taniguchi, and K. Oda, Radiat. Meas. 31, 261 (1999).

${ }^{16}$ R. D. H. Chu, G. Van Dyk, D. F. Lewis, K. P. J. O'Hara, B. W. Buckland, and F. Dinelle, Radiat. Phys. Chem. 35, 767 (1990).

${ }^{17}$ J. Fuchs, P. Antici, E. d'Humieres, E. Lefebvre, M. Borghesi, E. Brambrink, C. A. Cecchetti, M. Kaluza, V. Malka, M. Manclossi, S. Meyroneinc, P. Mora, J. Schreiber, T. Toncian, H. Pepin, and P. Audebert, Nat. Phys. 2, 48 (2006).

${ }^{18}$ M. Hegelich, S. Karsch, G. Pretzler, D. Habs, K. Witte, W. Guenther, M. Allen, A. Blazevic, J. Fuchs, J. C. Gauthier, M. Geissel, P. Audebert, T. Cowan, and M. Roth, Phys. Rev. Lett. 89, 085002 (2002).

${ }^{19}$ M. Zepf, E. L. Clark, F. N. Beg, R. J. Clarke, A. E. Dangor, A. Gopal, K. Krushelnick, P. A. Norreys, M. Tatarakis, U. Wagner, and M. S. Wei, Phys. Rev. Lett. 90, 064801 (2003).

${ }^{20}$ J. H. de Boer and F. A. Baker, Vacuum 16, 309 (1966).

${ }^{21}$ L. Changgeng, W. Yongqiang, Y. Shengsheng, J. Hui, and Z. Zhihao, Vacuum 46, 295 (1995)

${ }^{22}$ V. Kh. Alimov and V. N. Chernikov, J. Nucl. Mater. 273, 277 (1999). 